\title{
Are Italian Pulmonologists aware of the Guidelines for asthma management and do they know how to apply them?
}

\author{
E. Bacci1, L. Melosini1, F. Novelli1, S. Marinaro1, A.P. Pala², \\ A. Angino2, F.L. Dente1, P. Paggiaro1
}

ABSTRACT: Are Italian Pulmonologists aware of the Guidelines for asthma management and do they know how to apply them? E. Bacci, L. Melosini, F. Novelli, S. Marinaro, A.P. Pala, A. Angino, F.L. Dente, P. Paggiaro.

Background. Since 1995 GINA (Global Initiative on Asthma) guidelines for asthma management have been updated annually and published in order to promote better management of asthma in real life situations. The aim of our study was to assess the level of implementation of GINA Guidelines among Italian Pulmonary Specialists (PSs).

Methods. A detailed questionnaire was sent to 296 Respiratory Units (RUs) in Italy in order to collect information about personnel involved in the management of asthma patients, availability and use of diagnostic tools, recommended treatment according to the degree of asthma severity, educational activity. Data were analysed by using the SPSS programme.
Results. 74 (25\%) questionnaires were returned and analysed. Most RUs $(\mathbf{7 0} \%)$ do not have a dedicated asthma clinic; however, spirometry is available in more than $\mathbf{9 0 \%}$ of RUs, although it is performed in no more than $50 \%$ of patients in most RUs. Asthma treatment concurs with GINA recommendations in most RUs. Educational activity is performed by almost all RUs, usually in informal manner, during clinical visits, whereas only few RUs arrange individual educational sessions or "asthma school".

Conclusions. GINA guidelines for asthma management are applied by most Italian RUs included in this study in regard to educational activity and, to a lesser extent, to treatment. Surprisingly, many RUs perform spirometry in a relatively small number of patients despite its availability.

Monaldi Arch Chest Dis 2011; 75: 2, 120-125.

1 Cardio-Thoracic and Vascular Department, University of Pisa,

2 CNR Institute of Clinical Physiology, Pulmonary Environmental Epidemiology Unit, Pisa, Italy.

Correspondence: Dott.ssa Elena Bacci, Dipartimento Cardiotoracico e Vascolare, Via Paradisa 2, 56124 Pisa, Italy; e-mail: elena.bacci@gmail.com

The study was supported by a research grant from the Italian Agency of Drugs (AIFA) (progetto ARGA 2006, FARM5JYS5A).

\section{Background}

Bronchial asthma is one of the most frequent chronic respiratory diseases, affecting up to $5 \%$ of the general population in European countries, mainly children and elderly people [1]. The prevalence of asthma is still increasing, particularly in children and young adults [1], due to the persistence of the most common risk factors for asthma, such as atopic predisposition, exposure to indoor and outdoor allergens (including occupational sensitisers) and pollutants, and viral infections [2]. The increase in asthma mortality during the $1980 \mathrm{~s}$ led the National Heart, Lung \& Blood Institute (NHLBI) to consider asthma as "out of control" and promote initiatives in order to reduce the burden of asthma on the health system. The first document on recommendations for asthma management was published in 1995 [3]. After that period, asthma guidelines promoted by NHLBI (Global Initiative for Asthma, GINA) have been published and updated annually. The latest document was published in December 2009 [4], with continuous updating according to the results of the main studies published and the progression of knowledge regarding the management of this disease.

GINA guidelines aim to promote better management of asthma in real life situations, taking into account local differences worldwide. For this reason, a regional/national adaptation of these guidelines has been strongly recommended, and some countries have produced local versions of GINA Guidelines. In Italy, a local version of GINA guidelines, which takes into account specific Italian health organisation issues and the opinion of the main Italian researchers in the field, has been produced and disseminated in the last 10 years. This document is updated annually [5] and includes recommendations for correct diagnosis, assessment and management of asthma in Italy.

In the last years, a big effort has been made to disseminate asthma guidelines among pulmonary specialists (PSs) and general practitioners (GPs) in Italy. However, the real impact of the dissemination of GINA Guidelines on current clinical practice is unknown. Despite the formal declaration of 
a good knowledge of GINA Guidelines by PSs and GPs [6], the implementation of GINA Guidelines in the clinical practice is still poor. Very few surveys have been performed among PSs, in order to assess whether they are used to managing asthmatic patients according to national and international recommendations [7].

The real level of asthma control in general practice has been demonstrated by several surveys performed in some unselected samples of general population. All these studies, including the most recent ones [8], confirm that asthma control in Italy is still poor: more than $50 \%$ of asthmatic subjects have uncontrolled asthma, as assessed by the evaluation of daily symptoms, use of rescue medication, rate of exacerbations, and limitations in daily life. This might be due to the fact that a large number of asthmatic subjects do not seek medical assistance, do not undergo PS evaluation and may thus be under-treated.

The aim of this study was to assess the level of implementation of GINA Guidelines among Italian Pulmonary Specialists (PSs). We planned a cross-sectional survey among the Respiratory Units in the Italian Hospitals, in order to assess how asthma is really managed, by means of a questionnaire concerning arrangement of activity, availability of diagnostic tools and management of patients.

\section{Methods}

A detailed questionnaire was prepared and sent to each Respiratory Unit (RUs) of all Italian Hospitals and University Medical Centres. The questionnaire included information about the following issues: a) data about the arrangement of outpatient activity and the specific personnel dedicated to asthma management; b) type of investigations performed, and in which percentages of asthmatic patients; c) methods commonly used for the diagnosis of asthma; d) distribution of asthma severity of patients attending to the RUs; e) the rate of followup visits; f) type of drug therapy more frequently recommended (including specific immunotherapy); g) real implementation of educational activity on asthma. A copy of the questionnaire (Italian version) is shown in Appendix 1.

The list of all Respiratory Units in Italy was obtained from several sources: databases of the two main Italian Respiratory Societies (Associazione Italiana Pneumologi Ospedalieri - AIPO, and Società Italiana di Medicina Respiratoria SIMER), databases of major Italian pharmaceutical companies, and directly from many Italian opinion leaders in asthma. Because of several discrepancies between "official" databases and current information available, all regional delegates of the main Respiratory societies (AIPO and SIMER) were requested to provide a detailed list of all Respiratory Units in their region. Thereafter, a comprehensive, exhaustive list of all Respiratory Hospital Units in Italy was compiled.

Questionnaires were mailed to all Italian Respiratory Units; after the first mailing, 60 out of 247 questionnaires were completed and returned to our centre. A second mailing was performed to all Units that had not replied to the first mailing, and only 5 more questionnaires were completed and returned. A third attempt was made by e-mailing an electronic version of the same questionnaire to all the remaining Units whose email address was available $(n=31)$. In the meantime, information regarding 138 more Respiratory Units had been collected, so the online version of the questionnaire was also sent to those Units, in the latter group, whose email address was available $(n=49)$. Only 9 Units completed the online questionnaire. Thus, the questionnaire was eventually completed and returned by 74 of the 296 Units that had received it (total response rate: $25 \%$ ).

The completed questionnaires were transferred to an electronic database and statistically analysed by using the SPSS program. The distribution of responses to each question have been reported as number of responses; some continuous variables have been reported as mean and standard deviation, median and range or mode and range. Sub analyses according to the dimensions and other characteristics of Respiratory Units have been performed.

\section{Results}

Data from 74 questionnaires were analysed. They had been completed and returned by 38 Respiratory Units (RUs) in Northern Italy, 12 in Central Italy and 24 in Southern Italy. According to the questionnaires, most Units (70\%) do not have a dedicated asthma clinic. Most (46 out of 74) are "Complex Pulmonary Units" whereas only 8 are "Out-patient Clinic Units". The median number of physicians per RU is 5 (range: 1-17) and most are involved in asthma management. The most frequently reported number of nurses dedicated to the out-patient clinic is about 2 (range: $0-15$ ), all also involved in asthma management. Twenty-two RUs have a dedicated outpatient asthma clinic.

The median number of asthmatic subjects examined in one month is 14 , with wide variation (figure 1), whereas the median number of new asthma cases in one month is 3.5 (range: $0.5-20$ ). Two thirds of the RUs currently visit less than 20 asthmatic patients in one month, and the number of new cases of asthma observed in the out-patient clinic is less than 5 patients/month in most RUs. More than $90 \%$ of RUs have facilities allowing partial (Vital Capacity and Flow-Volume Curve) and full spirometry (including static lung volumes and diffusing capacity), and methacholine challenge test. More than $80 \%$ of RUs perform skin prick tests and Peak Expiratory Flow (PEF) monitoring. The number of spirometries performed in one week is high (median value: 40 tests, with a large variation, up to 800 in one Unit), but more than $80 \%$ of RUs perform spirometry in no more than $50 \%$ of asthmatic patients (figure 2); methacholine challenge test is less frequently performed (median number of tests per week: 5 , range: 0-50); skin prick test is performed in a greater number of 


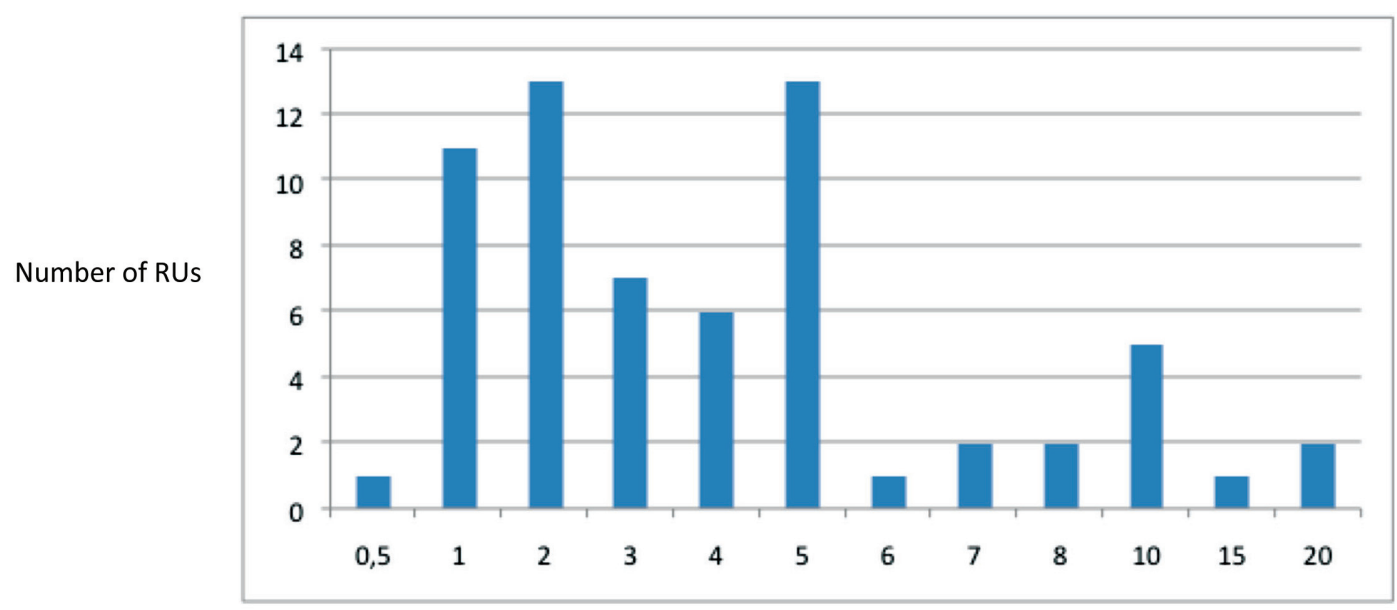

Number of new cases

Fig. 1. - New asthma cases per month.

patients (median number of tests per week: 12, range: 0-180). The measurement of exhaled nitric oxide (eNO) is available in 11 centres only, and in 10 of them it is measured in more than $80 \%$ of asthmatic patients; sputum analysis is performed in 8 centres only, and in 6 of them it is used in more than $50 \%$ of asthmatic patients. Thus, biomarkers are assessed in a minority of centres, but, when available, they are used in relatively high percentages of patients.

Asthma is diagnosed according to current Guidelines. Indeed, asthma diagnosis based only on clinical data is made in less than $20 \%$ of patients in most RUs, whereas other measurements (such as skin prick tests or PEF monitoring or the use of biomarkers) are used in a minority of RUs (table 1).
The distribution of asthma severity is reported in table 2. Most RUs report that 20-50\% of patients are mild or moderate persistent asthmatics, whereas less than $20 \%$ of patients are severe asthmatics. A large number of RUs declare to observe high percentages of patients with intermittent asthma.

Most RUs recommend the first follow-up visit after 1-3 months in more than $50 \%$ of patients, whereas only a minority of RUs leave the patients free to decide whether and when to request followup visits.

The pharmacologic therapy recommended by the different RUs is reported in table 3. Monotherapy with inhaled corticosteroids (ICS) or leukotriene receptor antagonists (LTRA) is recommended in less than $40 \%$ and $20 \%$ of the asthmatic patients respectively in almost all RUs. Surpris-

Number of RUs

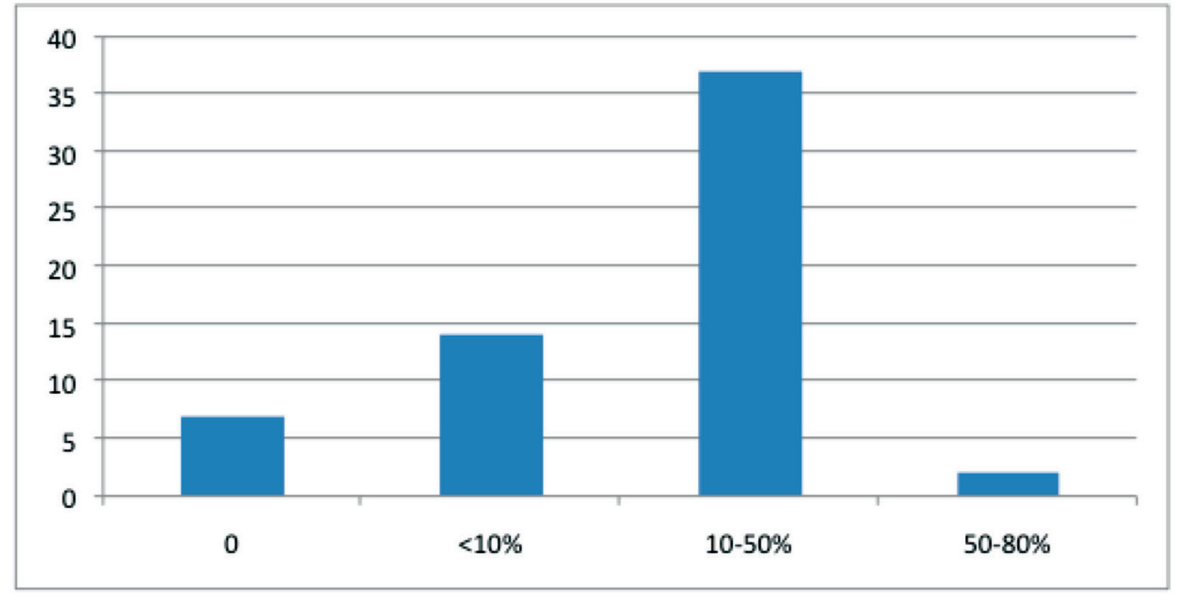

Number of new cases

Fig. 2. - Spirometries $(\mathrm{FVC}+\mathrm{VC})$ performed in asthma patients. 
Table 1. - Number of RUs performing asthma diagnosis by using the different types of evaluation in different percentages of patients

\begin{tabular}{lcccc}
\hline & $<\mathbf{2 0 \%}$ & $\mathbf{2 0 - 5 0 \%}$ & $\mathbf{5 0 - 8 0 \%}$ & $>\mathbf{8 0 \%}$ \\
\hline Clinical diagnosis & 62 & 11 & 1 & 0 \\
+ skin prick tests & 50 & 20 & 3 & 0 \\
+ PEF monitoring & 63 & 9 & 0 & 0 \\
+ biomarkers & 68 & 2 & 0 & 0 \\
\hline
\end{tabular}

Table 2. - Number of RUs reporting the percentage of patients for each level of severity

\begin{tabular}{lcccc}
\hline & $<\mathbf{2 0 \%}$ & $\mathbf{2 0 - 5 0 \%}$ & $\mathbf{5 0 - 8 0 \%}$ & $\mathbf{8 0 \%}$ \\
\hline Intermittent & 34 & 36 & 1 & 0 \\
Mild persistent & 14 & 54 & 3 & 0 \\
Moderate persistent & 7 & 56 & 8 & 0 \\
Severe, controlled & 49 & 22 & 0 & 0 \\
Severe, uncontrolled & 69 & 2 & 0 & 0 \\
\hline
\end{tabular}

ingly, a great number of RUs (45\%) recommend long-acting beta2-agonists (LABA) monotherapy, although most of them in less than $20 \%$ of asthmatic patients. As expected, most RUs recommend ICS+LABA combination in more than $40 \%$ of asthmatics, whereas ICS+LTRA combination is recommended in a smaller percentage of patients. Less than $20 \%$ of asthmatic patients need longterm treatment with systemic CS, whereas the use of injected depot CS is not recommended at all.

Most RUs recommend other treatments, such as antihistamines, oral theophylline, anti-IgE monoclonal antibodies and cromones, in a small number (less than 20\%) of asthmatic patients. Only few RUs recommend specific immunotherapy in high percentages (up to $60 \%$ ) of patients, whereas one third of RUs do not recommend it at all; this may be, at least in part, because the costs of immunotherapy are fully charged on patients in most Italian Regions [9].

Asthma control is assessed according to clinical evaluation, often including spirometry, whereas most RUs use PEF monitoring or biomarkers in a very small number of patients (table 4 ).
Educational activity is performed by almost all RUs, usually in informal way, during clinic visits (table 5). Few RUs arrange individual educational sessions or "asthma school", whereas most RUs distribute educational booklets and plan intervention for smoking cessation in currently smoking asthmatic patients. Compliance to asthma treatment is regularly assessed, although without using objective methods, and the correct use of the inhalers is also regularly checked at each visit in most RUs.

\section{Discussion}

By using different approaches, we demonstrated that Asthma Guidelines are largely applied in clinical practice by most PSs included in this study and that treating asthmatic patients according to Guidelines results in good control of asthma.

The data obtained from the questionnaire on the management of asthma show that most PSs from this selected sample of RUs in Italy are currently, albeit sometimes only partly, applying Guidelines for asthma diagnosis and treatment. In

Table 3. - Number of RUs recommending the different drug categories in different percentages of patients

\begin{tabular}{lcccccc}
\hline & $\mathbf{0 \%}$ & $\mathbf{< 2 0}$ & $\mathbf{2 0 - 4 0 \%}$ & $\mathbf{4 0 - 6 0 \%}$ & $\mathbf{6 0 - 8 0 \%}$ & $\mathbf{8 0 - 1 0 0 \%}$ \\
\hline Only ICS & 7 & 33 & 28 & 3 & 0 & 0 \\
Only LABA & 41 & 17 & 2 & 10 & 0 & 0 \\
Only LTRA & 35 & 28 & 2 & 0 & 0 & 0 \\
LABA + ICS & 0 & 1 & 11 & 18 & 35 & 9 \\
LABA + LTRA & 25 & 33 & 4 & 4 & 0 & 0 \\
LTRA + ICS & 14 & 43 & 5 & 2 & 1 & 0 \\
Systemic CS $>$ mos/year & 25 & 46 & 1 & 1 & 0 & 0 \\
Depot CS & 71 & 1 & 0 & 0 & 0 & 0 \\
\hline
\end{tabular}


Table 4. - Number of RUs using the different methods of assessing the level of asthma control in different percentages of patients

\begin{tabular}{lcccc}
\hline & $\mathbf{< 2 0 \%}$ & $\mathbf{2 0 - 5 0 \%}$ & $\mathbf{5 0 - 8 0 \%}$ & $>\mathbf{8 0 \%}$ \\
\hline Clinical assessment* & 15 & 20 & 14 & 23 \\
+ spirometry & 5 & 26 & 18 & 24 \\
+ PEF monitoring & 42 & 23 & 3 & 2 \\
+ biomarkers & 60 & 6 & 3 & 1 \\
\hline
\end{tabular}

* Symptoms, exacerbations, limitation in daily life.

Table 5. - Number of RUs performing different types of educational activity (EA)

\begin{tabular}{lccc}
\hline & Yes & No & Missing \\
\hline Is EA performed? & 68 & 6 & - \\
Informal, during the visit & 60 & 2 & 12 \\
Individual, with dedicated personnel & 17 & 30 & 27 \\
In groups (“Asthma School”) & 9 & 36 & 27 \\
Educational booklets & 61 & 12 & 1 \\
Smoking cessation center available & 56 & 15 & 3 \\
\hline
\end{tabular}

particular, the diagnosis of asthma is correctly based on clinical and functional evaluation, but many RUs perform spirometry in less than $50 \%$ of asthmatic patients, whereas methacholine challenge test is even less frequently used and biomakers are not used in current practice. Similar comments can be made for the assessment of asthma control. With regard to the type of treatment, most RUs recommend the use of ICS/LABA combination in large percentages of their asthmatic patients; however, a high percentage of RUs recommend LABA monotherapy, though in small percentages of asthmatic patients. Although the number of patients potentially treated with LABA alone seems fairly small, this is in complete disagreement with both international guidelines and Italian Agency of Drugs (AIFA) recommendations, which strongly state that LABA monotherapy should never be prescribed due to the risk of severe adverse events.

Surprisingly, the rate of educational activity is high in most RUs, with large implementation of patient-physician interaction and often formal education on asthma. The organisation of RUs in terms of personnel and instruments available for asthma management seems largely sufficient.

Two major problems were observed in this study: a) it was very difficult to obtain a complete and reliable list of all RUs in Italy; indeed, no list from the archives of the main scientific Italian Respiratory societies was exhaustive, so that we had to compare and cross-check information obtained from different sources; the result of this effort has led to a fairly comprehensive list of all possible RUs in Italy; b) the very low rate of reply from the different RUs (about 25\%), which may be explained by several reasons (wrong postal or email address, lack of interest in the initiative, etc.) may have selected the RUs with greater interest and motivation on asthma management. Thus, the data obtained may overestimate the rate of implementation of asthma Guidelines among RUs. This result is in disagreement with available literature data showing that guidelines are largely disregarded. Indeed, over $10 \%$ doctors ignore the existence of $78 \%$ of available guidelines [10]. This discrepancy might be due to differences in study population: most studies deal with general practitioners (GPs), who may have less chance to get acquainted with guidelines concerning many different topics. However, one meta-analysis published in the late 90 s showed that up to $84 \%$ of specialists were not aware of guidelines related to their practice [11]; such results might reflect a poor effort spent in disseminating guidelines, which has instead been increasing in the last decade. In addition, doctors may interpret guidelines as an excessive simplification, or something that inhibits their autonomy and flexibility and make the doctor-patient relationship impersonal [10].

In conclusion, the knowledge and the implementation of the asthma guidelines among Italian Pulmonary Specialists seems fairly high, although a positive selection bias may have overestimated this conclusion. This observation may support the hypothesis that other problems related to the perception of the patient and the complexity of the disease may explain the current poor control of asthma in Italy.

\section{References}

1. Eder W, Ege MJ, von Mutius E. The asthma epidemic. N Engl J Med 2006; 355: 2226-35.

2. Le Souëf PN. Gene-environmental interaction in the development of atopic asthma: new developments. Curr Opin Allergy Clin Immunol 2009; 9: 123-7. 
3. National Institutes of Health, NHLBI. Global Strategy for Asthma Management and Prevention. NHLBI/WHO Workshop report, NIH publication No. 95-3659, January 1995.

4. Global Initiative for Asthma www.ginasthma.org, december 2009 update.

5. Progetto Italiano LIBRA, www.ginasma.it, aggiornamento 2009.

6. Canonica GW, Baena-Cagnani C, Blaiss MS, et al. Unmet needs in asthma: Global asthma Physician and Patient (GAPP) survey: global adult findings. Allergy 2007; 62: 668-674.

7. Doerschug KC, Peterson MW, Dayton CS, Kline JN. Asthma guidelines: an assessment of physician understanding and practice. Am J Respir Crit Care Med 1999; 159: 1735-41.

8. Demoly P, Paggiaro P, Plaza V, et al. Prevalence of asthma control among adults in France, Germany, Italy, Spain and UK. Eur Respir Rev 2009; 18: 112, 105-111.

9. Tozzi AE, Armenio L, Bernardini R, et al. Pediatric allergy and immunology in Italy. Pediatr Allergy Immunol 2011; 22: 267-276.

10. Baiardini I, Braido F, Bonini M, Compalati E, Canonica GW. Why do doctors and patients not follow guidelines? Curr Opin Allergy Clin Immunol 2009; 9: 228-33.

11. Cabana MD, Rand CS, Powe NR, Wu AW, Wilson $\mathrm{MH}$, Abboud PA, Rubin HR. Why don't physicians follow clinical practice guidelines? A framework for improvement. JAMA 1999; 282: 1458-65.

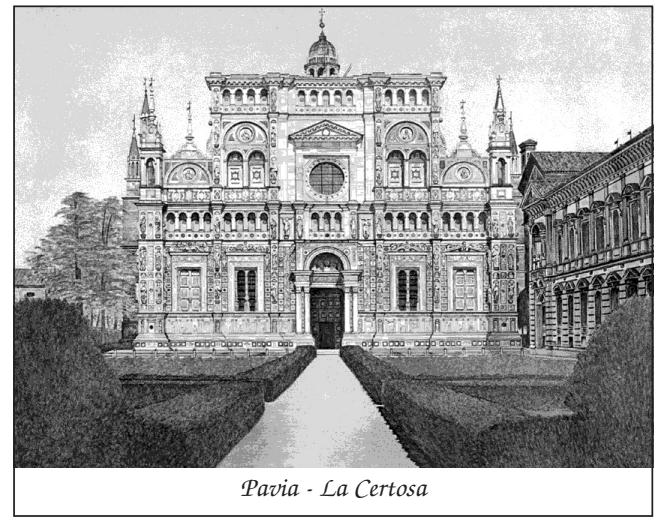

